\title{
The Totally Active Scintillator Detector
}

\author{
Alan Bross ${ }^{12}$ \\ Fermi National Accelerator Laboratory \\ Batavia, IL 60510-0500, USA \\ E-mail: bross@fnal.gov \\ Malcolm Ellis \\ Brunel University \\ Uxbridge, Middlesex UB8 3PH, United Kingdom \\ E-mail:malcolm.ellis@brunel.ac.uk
}

\section{Steve Geer}

Fermi National Accelerator Laboratory

Batavia, IL 60510-0500, USA

E-mail: sgeer@fnal.gov

\section{Enrique Fernandez-Martinez}

Max Planck

Germany

E-mail:enfmarti@mppmu.mpq.de

\section{Olga Mena}

INFN - Sez. Di Roma,Dipartimento di Fisica, Universit'a di Roma

P.le A. Moro, 5, I-00185 Roma, Italy

E-mail:olga.menarequejo@roma1.infn.it

\section{Silvia Pascoli}

IPPP, Department of Physics, Durham University

Durham DH1 3LE, United Kingdom

E-mail:silvia.pascoli@durham.ac.uk

For baselines of $\mathrm{O}(1000 \mathrm{~km})$, the rich oscillation pattern at a Low-Energy Neutrino Factory $\left(\mathrm{E}_{v}=0.5-\approx\right.$ $3 \mathrm{GeV}$ ) provides unique performance with regard to sensitivity to $\mathrm{CP}$ violation and the determination of the neutrino mass hierarchy [1]. A unique neutrino detector is needed, however, in order to exploit this oscillation pattern. The Totally Active Scintillator Detector (TASD) is particularly well suited for this facility. In this paper we give an update on the performance of TASD and discuss the detector R\&D program.

10th International Workshop on Neutrino Factories, Super beams and Beta beams

Valencia, Spain

30 June-05 July, 2008

\footnotetext{
${ }^{1}$ Speaker

${ }^{2}$ This work was supported by the Fermi National Accelerator Laboratory, which is operated by Universities Research Association, under contract No. DE-AC02-76CH03000 with the U.S. Department of Energy,
} 


\section{Introduction}

At baselines on the order of $1000 \mathrm{~km}$, the $4 \mathrm{GeV}\left(\mathrm{E}_{\mathrm{v}}=0.5-\approx 3 \mathrm{GeV}\right)$ Low-Energy Neutrino Factory (LENF) provides great sensitivity to the full spectrum of neutrino oscillation parameters. This is possible if the detector can fully exploit the rich oscillation pattern (see Figure 1) possible at this baseline and neutrino energy. The LENF requires a detector technology that can detect lower energy muons. Recently ideas have emerged for a Neutrino Factory detector based on a fully active calorimeter within a potentially affordable large volume magnet [2].

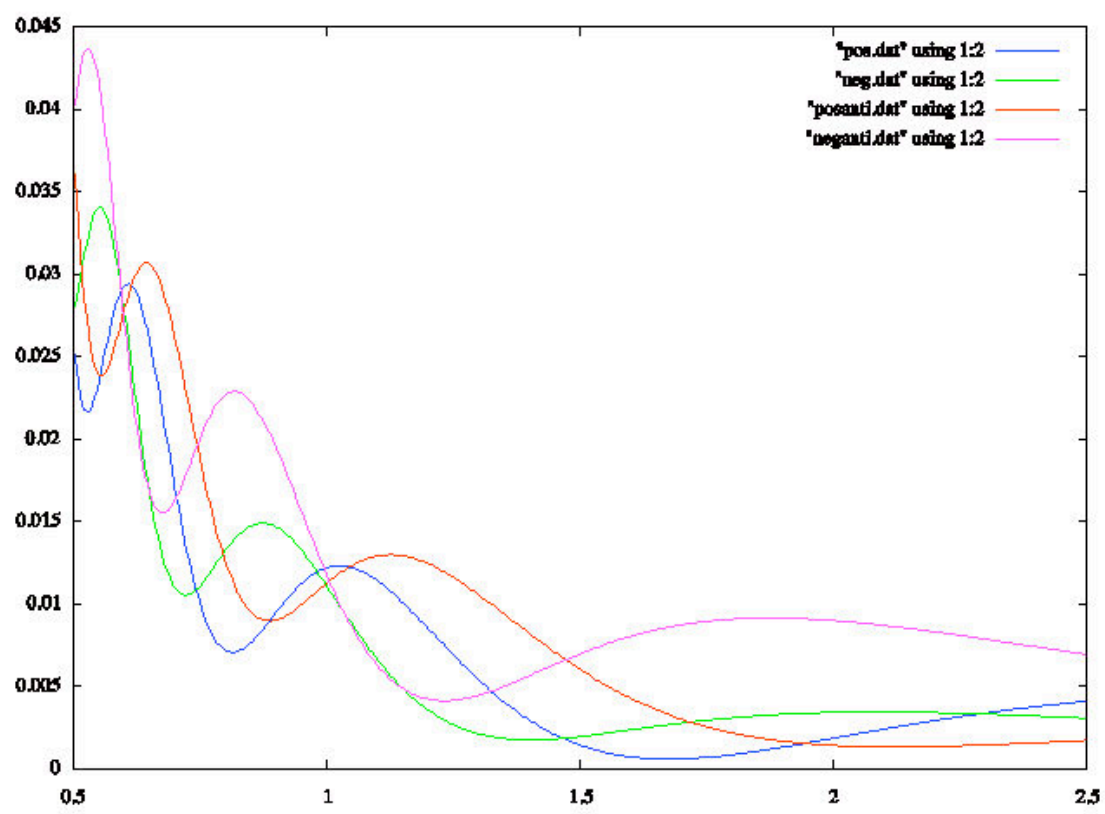

Figure 1 Oscillation Pattern as a function of neutrino energy for neutrinos and anti-neutrinos for normal and inverted hierarchy

\section{TASD}

The Totally Active Scintillator Detector consists of roughly 10,000 modules of triangular scintillator extrusions $\left(10^{7}\right.$ channels $)$ which are immersed in a $0.5 \mathrm{~T}$ magnetic field. The total detector mass is approximately $30 \mathrm{kT}$ with a fiducial mass of $20 \mathrm{kT}$.

\subsection{TASD Tracking Performance}

The detector has been simulated with GEANT4. The model included individual scintillator strips, and the digitisation assumed a readout performance based on measurements made with prototype scintillator for the MINERvA experiment [3]. A uniform 0.5T magnetic field was used for the simulation and reconstruction. Individual muons were simulated with initial momentum between $100 \mathrm{MeV} / \mathrm{c}$ and $5 \mathrm{GeV} / \mathrm{c}$. Monte Carlo truth information was used to aid the pattern recognition and the resulting set of space points was passed to a Kalman Filter [4] to fit the track. The reconstructed position resolution was determined to be approximately $4.5 \mathrm{~mm}$ 
RMS. The charge misidentification rate was determined by fitting each track twice, once with the correct charge and again with the incorrect charge. The misidentification rate was determined by counting the number of tracks in which the fit made with the incorrect sign had a better $\chi^{2}$ per degree of freedom than that made with the correct sign. The charge misidentification rate was approximately $2-3 \times 10^{-5}$ for muon momenta greater than $400 \mathrm{MeV} / \mathrm{c}$. Neutrino interactions were generated with the NUANCE code [5] and input into the GEANT4 simulation of the TASD in order to study the detector efficiency for charged current interactions. The reconstruction efficiency for $v_{\mu}$ charged current events is high above neutrino energy of $500 \mathrm{MeV}$, reaching a plateau of approximately $98 \%$ for neutrino interaction energy > $2 \mathrm{GeV}$. The charge mis-identification rate, neutrino reconstruction efficiency and neutrino energy resolution from the simulations were used to determine the physics reach of the TASD at a low energy neutrino factory [2]. This analysis showed that the combination of the low energy neutrino factory with the low energy threshold TASD is a powerful tool for studying neutrino oscillations through the golden channel.

\subsection{TASD Electron ID Capability}

In order to study the potential for the TASD to analyze electron neutrino interactions, and hence access the platinum channel, simulations were made of isolated positrons, with initial momenta ranging between $100 \mathrm{MeV} / \mathrm{c}$ and $4.9 \mathrm{GeV} / \mathrm{c}$. In this study the point reconstruction was simulated by randomly discarding hits to simulate the point reconstruction efficiency and then smearing the remaining hits with the position resolution determined from the muon studies. These smeared points were then presented in the form of an event display which showed the position perpendicular to the magnetic field (see Figure 2).
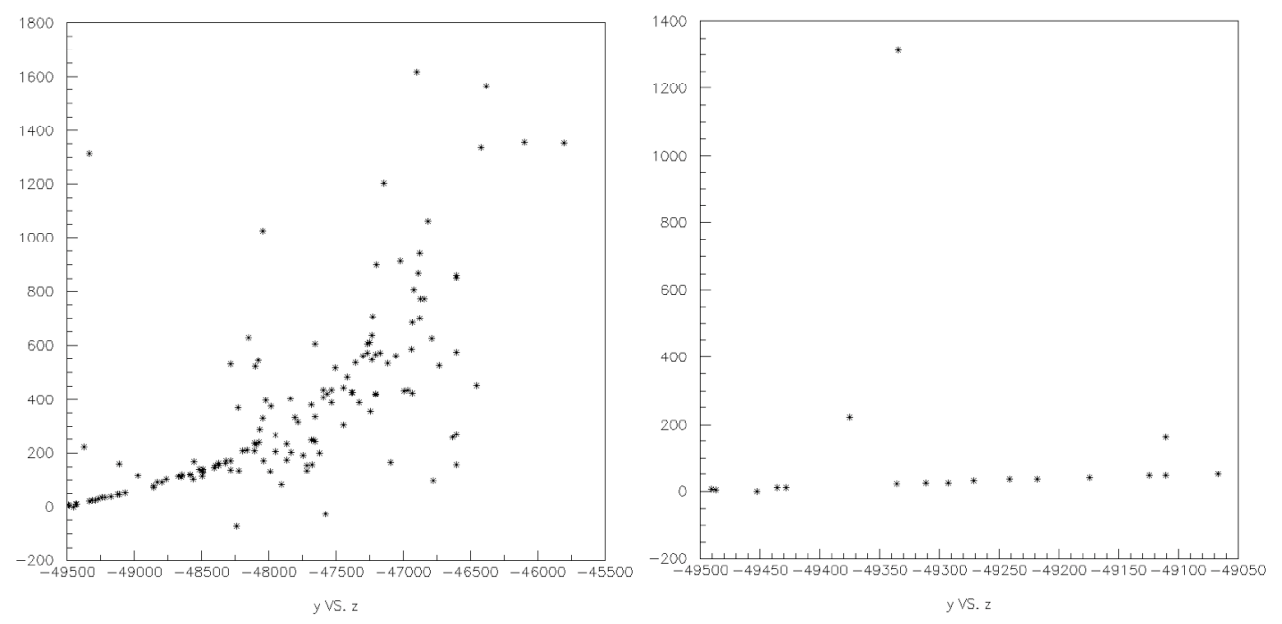

Figure 2 An example of the event display produced from the smeared Monte Carlo hits for a positron. The initial momentum was $2.4 \mathrm{GeV} / \mathrm{c}$ and despite the straight track seen in the zoomed view of the start of the track (right), it was still possible to correctly determine the direction that the particle bent by looking at the complete event (left).

The events were passed through a "blinder" program which randomly flipped the $\mathrm{Y}$ axis in the plot and distributed the different momentum bins randomly throughout the range of event numbers. In this way, it was not possible to determine the initial momentum or direction of curvature from the name of the image files. Each of the simulated events was studied independently by four people. They were told to look at the two images of each event and attempt to determine whether the initial particle was curving up or down. A third option of 
"unable to determine" could also be assigned to each event. In the vast majority of cases, everyone gave the same determination for each event. In rare cases, one person would disagree, in which case the determination for that event was taken as that produced by the majority of people.

In approximately $85 \%$ of the events, the scanners thought that they could determine the sign of the particle. It was often the case (see Figure 2) that while the stub appeared to be straight, the bending direction of the particle could be obtained from the downstream section of the track following a hard bremsstrahlung and thus and the correct charge assigned. Figure 3 (left) shows that the efficiency for making a determination for the sign from the scan did not depend strongly on the initial momentum.
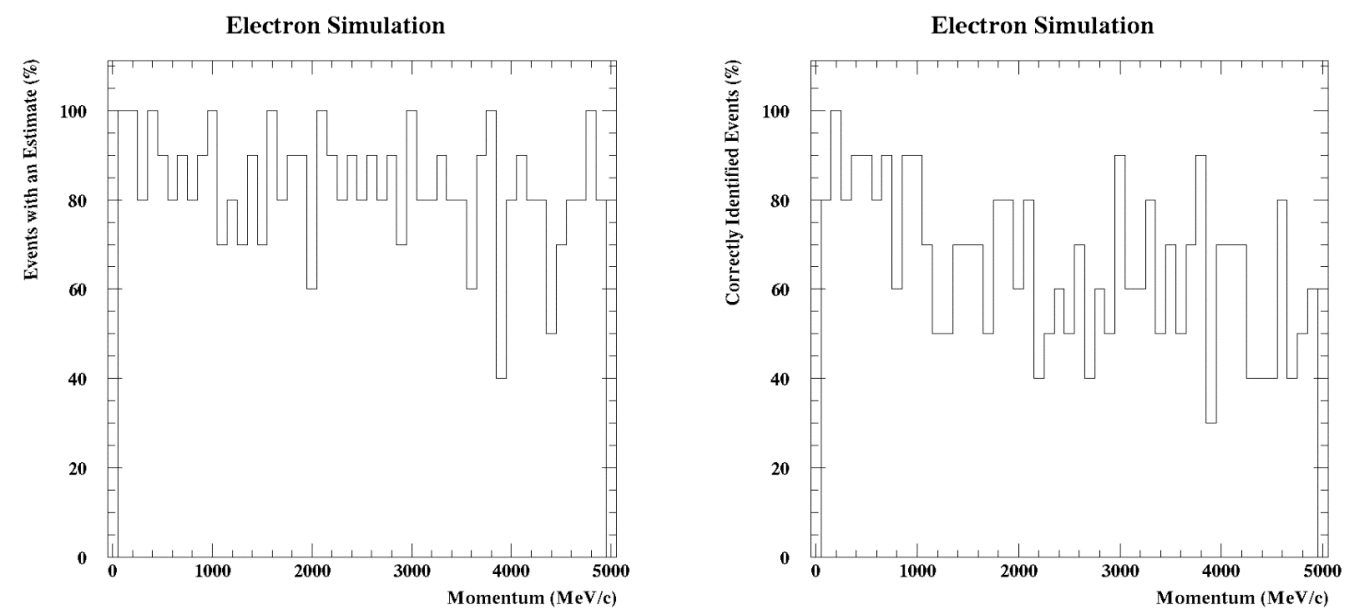

Figure 3 Fraction of events in which the sign could be determined versus the initial momentum (left), fraction of events in which the sign was determined correctly (right).

The charge assignment accuracy was determined by using the information produced by the blinding application to extract the true bending direction for each of the event display images.

Figure 3 (right) shows the fraction of events in which the charge was identified correctly. It should be noted that this ratio includes all the events for which no charge assignment could be made at all, and shows that at low momentum, almost all the charge assignments were correct. This very quick "by eye" reconstruction seems to be able to determine the charge of the track in approximately $80 \%$ of events. The charge is correctly identified almost all the time at low energy and even at $3 \mathrm{GeV} / \mathrm{c}$ only $30 \%$ of the events are given the wrong sign. The use of a proper track fit to the stub should allow these values to be improved.

\section{TASD R\&D Needs}

There are three key components to the TASD that require R\&D:

1. Magnet

2. Scintillator production

3. Photo-detector and electronics

The concept for the magnetisation of such a large volume is based around the superconducting transmission line (SCTL) developed for the VLHC project [6]. The SCTL consists of an outer vacuum jacket which provides thermal insulation, within which there is a liquid nitrogen shield and an inner pipe for liquid helium. Wrapped around the liquid helium pipe is a 
superconducting braid. The SCTL concept has been prototyped, tested and costed for the VLHC project at Fermilab.

A design for the TASD magnet using SCTL wrapped around a $15 \mathrm{~m}$ diameter mandrel that would house the TASD modules has been shown to require half the maximum current that was achieved for the VLHC SCTL and can generate an on-axis field of $0.6 \mathrm{~T}$ over most of the length of the detector. The basic concept of the scintillator requires a relatively small R\&D program to increase the through-put and efficiency for generating the scintillator. In addition a R\&D program to develop a reliable technique to co-extrude wavelength shifting fibre with the scintillator extrusion is envisioned. This technique has been successfully used on post-cladding Kuraray fiber with various polymers. However, in these tests, the post-clad material was much thinner than that which would be needed for a TASD element. The photo-detector and electronics R\&D is already occurring around the world. Hamamatsu, RMD and other companies are currently working on Silicon-PM devices (also known as MPPDs or MRSDs). The goal for the TASD would be to reduce the cost per channel to less than $\$ 5$.

\section{Conclusions}

A low energy Neutrino Factory, coupled with the right detector, gives excellent capability in exploring the full neutrino mixing matrix and measuring leptonic $\mathrm{CP}$ violation. A finely segmented TASD is quite possibly the right analysis tool for a low energy Neutrino Factory. Much more simulation and study are required; however the initial results are promising. The detector R\&D program required for the TASD is well defined and is dominated by the need to magnetise the large volume. A low energy $(4 \mathrm{GeV})$ Neutrino Factory is certainly cheaper than a $20 \mathrm{GeV}$ Neutrino Factory and with proper planning may even be upgradable to the high energy facility. The TASD performance appears to be impressive and while more study is required, it could even be useful for studying nucleon decay, atmospheric neutrinos and neutrinos from relic supernovae.

\section{References}

[1] A. Bross, M. Ellis, S. Geer, O. Mena, S. Pascoli, "A Neutrino Factory for both large and small theta(13)", Phys. Rev. D77:0933012, 2008.

[2] A. Bross, M. Ellis, S. Geer, O. Mena, S. Pascoli, "The low-energy neutrino factory", AIP Conf. Proc. 981:187-189,2008.

[3] A. Pla-Dalmau et al., "Extruded plastic scintillator for MINERvA", FERMILAB-CONF-05-506-E, Nov 2005.

[4] A. Cervera-Villanueva, J.J. Gomez-Cadenas, J.A. Hernando, "'RecPack' a reconstruction toolkit", Nucl. Instrum. Meth. A534:180-183, 2004.

[5] D. Casper, "The Nuance neutrino physics simulation, and the future", Nucl. Phys. Proc. Suppl. 112:161-170, 2002 .

[6] Ambrosio et al, Fermilab-TM-2149, June, 2001. 